\title{
Editorial: Ein merkwürdiger Fall
}

\author{
Rupert Gaderer \\ Ruhr-Universität Bochum, Germanistisches Institut, Universitätsstr. 150, \\ 44780 Bochum \\ rupert.gaderer@rub.de \\ Matthias Thiele \\ TU Dortmund, Fakultät Kulturwissenschaften, Emil-Figge-Str. 50, \\ 44227 Dortmund \\ matthias.thiele@udo.edu
}

Als Ingeborg Bachmann in einem Interview mit der Münchner Abendzeitung im Januar 1962 nach der sonderbaren Figur des Richters in der Erzählung Ein Wildermuth (1961) gefragt wurde, antwortete sie:

Als ich mich an der Universität einschrieb, hatte ich keine Ahnung, daß ich mich für eine Fakultät entscheiden müßte, so hospitierte ich denn zwei Semester gleichzeitig in der juristischen und philosophischen Fakultät. Das bedeutete, daß ich einen Sommer lang an einem Bezirksgericht praktizierte und mit aufs Land mußte, wenn Gerichtstag war. Das ist Pflicht für junge österreichische Jus-Studenten. ${ }^{1}$

Dass Bachmann im Sommersemester 1946 Philosophie, Germanistik und Jura in Graz studierte, ist der biographische Kontext der Erzählung über den Richter Anton Wildermuth, der einen merkwürdigen Fall darstellt: Während eines Gerichtsprozesses, bei dem über einen Mord verhandelt wird und ein Knopf ein wichtiges Beweisstück darstellt, stößt Wildermuth unvorhergesehen einen Schrei aus - woraufhin der Mordprozess unterbrochen und der Richter in die Obhut eines Nervenarztes gegeben wird. Nach dem Schrei verfasst der Richter eine Aufzeichnung über seine Suche nach der Wahrheit. Die Lehrformel dieser Suche ist die unerreichbare Aufforderung seines Vaters: „Ein Wildermuth wählt immer die Wahrheit.“2 Obgleich der biografische Aspekt interessant für das Genre des Interviews und die Verbindung von Leben und

1 Nöhbauer (1991 [1962]), S. 32.

2 Bachmann (2010 [1961]), S. 214. 
Schreiben ist, spielt er für die hier versammelten Beiträge eine untergeordnete Rolle. Das mag auch daran liegen, dass in der Rezeption von Bachmanns Werk biografische Lesarten oft in die Irre führten. Dennoch ist die ausgelegte Fährte der Autorin nicht einfach wegzuwischen, weil mit dem biografischen Verweis auf die Fakultäten in kalkulierter Weise Hauptthemen des eigenen Schreibens und der Erzählung adressiert werden: Literatur, Philosophie und Recht.

Bachmanns Erzählung, veröffentlicht im Erzählband Das dreißigste Jahr (1961), zählt sicherlich zu den von der Bachmann-Forschung vernachlässigten Werken der Autorin. ${ }^{3}$ Zwar wurden sprachphilosophische Fragen, vor allem vor dem Hintergrund der Auseinandersetzung Bachmanns mit Ludwig Wittgenstein und Martin Heidegger, ${ }^{4}$ an die Erzählung herangetragen, ${ }^{5}$ und es wurden ebenso erzähl- und gendertheoretische Aspekte beleuchtet; ${ }^{6}$ jedoch ließen die meisten Auseinandersetzungen den Zusammenhang zwischen Techniken der Wahrheitsfindung, Medien und Dingen des Rechts unberücksichtigt. Ausgehend von dieser Forschungslage besteht das Ziel des Themenheftes zunächst darin, die Verbindung zwischen Recht und Literatur zu untersuchen. Es ist erstaunlich, dass in der akribischen Erforschung der Romane, Erzählungen und Hörspiele diese Thematik nicht ausführlich erörtert wurde. Dabei bieten die Ergebnisse der Law-and-Literature- bzw. Law-as-Literature-Bewegung ${ }^{7}$ sowie die deutschsprachigen Forschungen zum Themenkomplex Recht und Literatur $^{8}$ einen stabilen Ausgangspunkt für derartige Analysen.

Bachmann setzte sich immer wieder mit dem Konnex von Recht und Literatur auseinander: angefangen bei den frühen Hörspielen Ein Geschäft mit Träumen (28.2.1952/RWR Wien) und Die Zikaden (25.3.1955/NWDR Hamburg), in denen legislatorische Themen und der Akt der Rechtsetzung behandelt werden; ${ }^{9}$ über die Erzählungen Der Tod wird kommen (1965) und Probleme Probleme (1972), in denen der Schwierigkeit nachgegangen wird, inwiefern juristische Strukturen des Urteilens ins private Zusammenleben der Familie eindringen und zur Belastung der Betroffenen werden; ${ }^{10}$ bis zu Drei Wege zum See (1972) und Malina (1971), in denen der Widerstand gegen Gesetze und das Grundrecht des Briefgeheimnisses thematisiert werden. Bezieht sich

3 Zu den einschlägigen Titeln der überschaubaren Sekundärliteratur zählen Höller u.a. (1987), S. 132-142; Töller (1998), S. 120-131; Philipp (2001), S. 99-111; Eberhardt (2002), S. 184-211.

4 Vgl. Agamben (1989).

$5 \quad$ Vgl. Solibakke (2012) u. Dietz (2003).

6 Vgl. Schneider (2013) u. Künzel (2007).

7 Vgl. Posner (1988) u. Lachenmaier (2008).

8 Vgl. u.a. Geulen (2012) u. Köhler/Müller-Mall/Schnädelbach (2017).

9 Vgl. Bachmann (2010 [1958]), S. 177-210; Bachmann (2010 [1958]), S. 217-268.

10 Vgl. Bachmann (2010 [1961]), S. 266-276; Bachmann (2010 [1961]), S. 318-353. 
diese kursorische Aufzählung von Beispielen auf die vielen Schnittpunkte von Literatur und Recht, so fällt auf, dass Bachmann mehrere Male den Akt des Rechtsprechens von den Rändern ins Zentrum ihres literarischen Erkenntnisinteresses rückte: Zwischen 1951 und 1953 verfasste Bachmann insgesamt 15 Skripte für die populäre Sendung Die Radiofamilie, in der immer wieder Szenen juristischer Entscheidungen eine wichtige Rolle spielen. ${ }^{11}$ Nicht zuletzt bekleidet der Familienvater Hans Floriani - wie der Richter Wildermuth - das Amt eines Oberlandesgerichtsrats. Neben diesen lange Zeit vergessenen Radioarbeiten seien in aller Kürze Bachmanns zwischen 1954 und 1955 als römische Korrespondentin verfassten Reportagen für das Radio Bremen erwähnt, in denen sie unter dem Pseudonym Ruth Keller über den aufsehenerregenden Kriminal-Fall Wilma Montesi im Italien der Nachkriegsjahre berichtete. ${ }^{12}$ Schließlich bildet das Dispositiv des Gerichtsverfahrens in Bachmanns wohl bekanntestem Hörspiel Der gute Gott von Manhattan (BR/NDR 29.5.1958) die Ausgangslage für die Verkettungen, Rekonstruktionen und das Erzählen über die Wahrheitsfindung eines Richters und eines selbstmächtigen Angeklagten. Bei diesen Beispielen sind Fragen nach Techniken der Wahrheitsfindung und dem (aufgeschobenen) Urteil zentrale Bestandteile von Bachmanns Auseinandersetzung mit der Medialität und den Kulturtechniken des Rechts.

Die Beiträger*innen des Themenheftes erweitern die für Bachmann grundlegende und wichtige Frage nach den Medien der Literatur um Ansätze der neueren Kulturtechnikforschung. ${ }^{13}$ Gerade Bachmanns Ein Wildermuth bietet sich für eine derartige Lektüre an, weil die Erzählung exemplarisch darauf aufmerksam macht, inwiefern Kulturtechniken dafür verantwortlich sind, dass Streit-Dinge in einem gerichtlichen Verfahren diskursiviert werden. ${ }^{14}$ Ermöglicht wird dieser potenzielle Bezug auf sich selbst durch die Rekursivität der Ausführungen von Kulturtechniken. ${ }^{15}$ Sie ermöglichen die Handlungsmacht von Dingen des Rechts und nehmen beim Trennen und Abwägen eine Position des Dritten ein, wenn sie eine Unterscheidung von Wahrheit und Lüge, Thema und Themenlosigkeit oderVernunft und Wahnsinn prozessieren. ${ }^{16}$ Die Kulturtechniken des Rechts sind gleichermaßen dafür verantwortlich, dass Macht ausgeübt, Recht vollzogen und Urteile gesprochen oder verschwiegen werden. Dies wird auch bei Bachmann kenntlich, wenn nach

\footnotetext{
11 Vgl. McVeigh (2012), S. 337-388.

12 Vgl. Bachmann (1998).

13 Vgl. zu medienwissenschaftlichen Ansätzen Weigel (1999) und Simons/Weber (2008).

14 Vgl. Vismann (2011).

15 Vgl. Siegert (2001) u. Schüttpelz (2006).

16 Vgl. Steinhauer (2014).
} 
den Kulturtechniken und Dingen des Rechts im Sinne eines ,Making of Law gefragt wird. ${ }^{17}$

Damit lädt die Erzählung dazu ein, über das Gericht hinaus die Wahrheitsbegriffe, das pädagogische Dispositiv der Wahrheit und die politische Praxis des Wahrsprechens - die parrhesia - zu reflektieren..$^{18}$ Relevant ist dabei, dass in der Erzählung das Geschehen auf der Gerichtssaalbühne in Kontrast zur Vertrautheit der Familienszene und dem Wahrheitsexzess von Vater und Sohn steht. Auch lässt sich von der Gerichtsszene ausgehend eine signifikante Verschiebung von Ein Wildermuth zu den späten Prosatexten Bachmanns erkennen, da letztere als Anklagetexte die Leserschaft selbst zu Gericht sitzen lassen. Da die narrative Konstruktion der Erzählung mit der poetischen Funktion der Übercodierung und dem Zufall, den Momenten des Wahrscheinlichen und des Notwendigen, arbeitet, um die Kondensate von ,Ich in der Geschichte und ,die Geschichte im Ich' zu reflektieren, ${ }^{19}$ kann das zeitliche Setting und die daran gebundene nationalsozialistische Vergangenheit des Vaters ermessen werden. Im Fokus solcher Lektüren, die die Erzählung mit dem diskursiven Komplex von Vergangenheitsaufarbeitung und Vergangenheitsentsorgung verknüpfen, gewinnt das kleine Detail des Knopfs als Streit-Ding vor Gericht an brisantem Gewicht für die Frage des Rechtsprechens.

Zudem drängt die theoretische Ausrichtung - Kulturtechniken, Medien und Recht - geradezu eine Lektüre des Textes auf, die vom Titel ausgeht. Der Titel stiftet nicht nur Recht und Gesetz, indem er dem Text im Ganzen Identität verleiht und ihn damit für jeglichen juristischen Kontext operabel macht. Er markiert auch die Grenzen des Textes nach Außen wie nach Innen und enthält zugleich ein Versprechen für die prozessuale Lektüre der Erzählung, die in Relation zum Titel mit einer Kippbewegung von Ent-Täuschen operiert. So widmet sich Armin Schäfer der Frage nach dem titelgebenden Namen der Erzählung und wählt dafür Bachmanns Frankfurter Vorlesungen (1959/60) als Ausgangspunkt seiner Überlegungen. Bachmanns Umgang mit und Überlegungen zu Namen werden als Ausdruck einer historischen Situation verstanden und mit Diskussionen über die mediale Figur der Übertragung sowie der Kontingenz und Bedeutung der Wahl verbunden. Relevant ist die Verbindung zwischen einer Logik der existenziellen Wahl und der Wahl des Namens, die Schäfer in Verbindung mit der Rolle von Wildermuths Vater und dessen Wahl der Wahrheit im Nationalsozialismus bringt. Zuletzt wird die väterliche Formel - „Ein Wildermuth wählt immer die Wahrheit.“ - als Aussage

17 Vgl. Latour (2010) u. Serres (1994).

18 Vgl. Foucault (1988; 2010).

19 Vgl. Göttsche (1998). 
herausgestellt, die einem autoritären Schema folgt, eine Wahrheit der Poesie ausschließt und zu Unterscheidungskriterien zwischen dem Diskurs des Rechts und der Literatur führt.

Für Annika Frank laden der Wechsel der Erzählperspektive und die komplexe Semantisierung der Erzählung dazu ein, die Sujets ,Gerichtsverhandlung ${ }^{\star}$ und ,Erzählen der eigenen Biografie‘ in linguistischer, funktional-pragmatischer Ausrichtung zu analysieren. Aufgezeigt werden die kontrastiven Folien der zwei Haupteile der Erzählung: zum einen die institutionalisierten Sprachhandlungen der Gerichtskommunikation, zum anderen das Diskursmuster autobiografischer Alltagserzählungen. Durch den Fokus auf Sprach- und institutionelle Sprechhandlungen werden sowohl das Verhältnis von Schriftlichkeit (Fallakten) und Stimme (Mündlichkeitsgrundsatz) als auch das von Zeugenaussage und Wissenstransfer als Grundierung des Wildermuth-Falls reflektiert.

In der Lektüre Thomas M. Seiberts ist der Schrei des Richters zentral. Der ehemalige Strafrichter liest Bachmanns Erzählung nicht in einer herkömmlichen literaturwissenschaftlichen Perspektive, sondern konzentriert sich auf eine rechtssemiotische Interpretation unter Einbezug der eigenen Arbeit als Richter. Dabei geht es ihm um die Frage nach der Konstruktion von Wahrheit in einem Strafprozess und jenem Umschlagspunkt, ab dem die Klarheit im Gerichtsverfahren zur obskuren Verschleierung der Tatsachen wird. Seibert setzt den Schrei des Richters in Relation zu Daniel Paul Schrebers Denkwürdigkeiten eines Nervenkranken (1903) und fragt danach, inwiefern die Sätze des Strafrechts auf die Wahrheit eines Falls passen. Der Schrei sprengt in dieser Lesart die juridische Form, weil er zutiefst antijuridisch ist.

Till Breyers Beitrag geht zunächst von Christa Wolfs Überlegungen zum Wahrheitsbegriff bei Bachmann aus, lässt diese mit sprachphilosophischen Überlegungen korrelieren, um in Abgrenzung dazu die Erzählung mit der Rechtspraxis in der Nachkriegsgesellschaft zu historisieren. Damit gelingt es ihm, die kritische Reflexion der geschichtlichen Verfasstheit des Rechtsprechens nach 1945 am Beispiel der Erzählung herauszuarbeiten. Der Beitrag hebt die Differenzierung von Monströsem und Normalem sowie die Destabilisierung des Rechts als Erzählanlass hervor.

Rupert Gaderer untersucht die Akteursbeschaffenheit von Dingen und die Vernetzungsleistung des Rechts in der Erzählung. Dabei arbeitet er heraus, inwiefern Bachmann ein epistemologisches Problem thematisiert, das in der Erzählung in unterschiedlichen Konstellationen befragt wird: Wie ist es möglich, einen Zugang zur (juristischen) Wahrheit zu erlangen, wenn es bereits außerordentlich schwierig ist, etwas über die Identität eines Knopfes auszusagen? Für Bachmann relevant sind literarische Diskurse über die Urteilskraft, 
die sie mit der Frage nach den Kulturtechniken und Dingen des Rechts verbindet. Die Suche nach der Wahrheit, wie sie der Richter Wildermuth nach dem Schrei aufzeichnet, wird getragen durch die Dispositive der Pädagogik, Bürokratie und Sexualität.

Matthias Thiele bezieht in seine Analyse mehrfach den Erzählband als Kontext von Ein Wildermuth mit ein. Aufgrund seiner Komposition und Vernetzung der Erzählungen wird in einem ersten Schritt die Parallelität von Ein Wildermuth und Das dreißigste Jahr untersucht, deren Themen und Motive sich in der Gemeinsamkeit bündeln, dass es sich in beiden Fällen um facettenreiche (De-)Normalisierungsnarrationen handelt. Ein Wildermuth hebt sich von Das dreißigste Jahr und allen weiteren Erzählungen des Bandes vor allem durch Komik ab. Entsprechend werden in einem zweiten Schritt die komischen Formen, Verfahren, Elemente und Segmente analysiert, wobei die Gerichtszene mit dem Knopfexperten den komödiantischen Höhepunkt darstellt. Die durch Kontrast und Inkongruenz erzeugten komischen Effekte dienen vor allem zur Distanzerzeugung und Vermeidung einer identifikatorischen Lektüre insbesondere bezüglich des zweiten Kapitels mit seiner geständnisartigen Narration.

Walter Grünzweig beschäftigt sich in seinem Beitrag damit, inwiefern Bachmann zum Kanon der Auslandsgermanistik hauptsächlich an den US-amerikanischen Universitäten im Kontext von Women Studies/Women Literature, World Literature und Austrian Literature Fiction gehört. So wurden auch der Erzählband Das dreißigste Jahr und die Erzählung Ein Wildermuth von dem Max Frisch-Übersetzer Michael Bullock ins Englische übertragen. Die Übersetzung wird als Interpretation semantischer Potenziale und auf semantisch relevante Auffälligkeiten hin analysiert. Durch einen komplexen, von Rückübersetzungen semantischer, Knoten' durchzogenen Dialog zwischen Original und Übersetzung wird aufgezeigt, dass die Kulturtechnik des Übersetzens nicht nur den interkulturellen Transfer von Texten und Literatur ermöglicht und sichert, sondern als übersetzerische Interpretationsleistung zugleich zu informierteren Neulektüren beiträgt.

Das Themenheft Bachmanns „Ein Wildermuth“. Kulturtechniken, Medien und Recht geht auf den gleichnamigen Workshop zurück, der von den Gastherausgebern im März 2018 an der TU Dortmund veranstaltet wurde. Das Themenheft setzt sich aus den verschriftlichten Vorträgen der Teilnehmer*innen des Workshops und weiteren Autor*innen zusammen. 


\section{Literaturverzeichnis}

Agamben, Giorgio (1989): „Il silenzio delle parole“, in: Christine Koschel/Inge von Weidenbaum (Hg.): Ingeborg Bachmann. In cerca di frasi vere. Bari u.a.: Giuseppe Laterza \& Figli, S. v-Xv.

Bachmann, Ingeborg (1998): Römische Reportagen. Eine Wiederentdeckung. Hg. von Jörg-Dieter Kogel. München: Piper.

Bachmann, Ingeborg (2010 [1958]): Die Zikaden, in dies.: Werke, Bd. 1, Gedichte, Hörspiele, Libretti, Übersetzungen. Hg. von Christine Koschel/Inge von Weidenbaum/ Clemens Münster. München/Zürich: Piper, S. 217-268.

Bachmann, Ingeborg (2010 [1958]): Ein Geschäft mit Träumen, in dies.: Werke, Bd. 1, Gedichte, Hörspiele, Libretti, Übersetzungen. Hg. von Christine Koschel/Inge von Weidenbaum/Clemens Münster. München/Zürich: Piper, S. 177-216.

Bachmann, Ingeborg (2010 [1961]): Probleme Probleme, in dies.: Werke, Bd. 2, Erzählungen. Hg. von Christine Koschel/Inge von Weidenbaum/Clemens Münster. München/Zürich: Piper, S. 318-353.

Bachmann, Ingeborg (2010 [1961]): Der Tod wird kommen, in dies.: Werke, Bd. 2, Erzählungen. Hg. von Christine Koschel/Inge von Weidenbaum/Clemens Münster. München/Zürich: Piper, S. 266-276.

Bachmann, Ingeborg (2010 [1961]): Ein Wildermuth, in dies.: Werke, Bd. 2, Erzählungen. Hg. von Christine Koschel/Inge von Weidenbaum/Clemens Münster. München/ Zürich: Piper, S. 214-252.

Eberhardt, Joachim (2002): „Es gibt für mich keine Zitate“: Intertextualität im dichterischen Werk Ingeborg Bachmanns. Tübingen: Max Niemeyer.

Foucault, Michel (1988): Das Wahrsprechen des Anderen. Zwei Vorlesungen von 1983/84. Hg. und eingeleitet von Ulrike Reuter u.a. Frankfurt a.M.: Materialis Verlag.

Foucault, Michel (2010): Der Mut zur Wahrheit. Die Regierung des Selbst und der anderen II. Vorlesung am Collège de France 1983/84. Berlin: Suhrkamp.

Geulen, Eva (2012): „Law and Literature. Who Owns It?“, in: Werner Gephart (Hg.): Rechtsanalyse als Kulturforschung. Frankfurt a.M.: Vittorio Klostermann, S. 309-322.

Göttsche, Dirk (1987): Die Produktivität der Sprachkrise in der modernen Prosa. Frankfurt a.M.: Athenäum.

Höller, Hans (1987): Ingeborg Bachmann. Das Werk. Von den frühesten Gedichten bis zum „Todesarten“-Zyklus. Frankfurt a.M.: Athenäum.

Köhler, Sigrid/Müller-Mall, Sabine/Schmidt, Florian/Schnädelbach, Sandra (2017): Recht fühlen. Paderborn: Fink.

Künzel, Christine (2007): „Aus einem Bette aufgestanden“ Anmerkungen zum ,Verhältnis‘ zwischen Recht und Literatur“, in: Gert Hofmann (Hg.): Figures of Law. Studies in the Interference of Law and Literature. Tübingen/Basel: A. Francke, S. 115-132. 
Lachenmaier, Birgit Maria (2008): Die Law as Literature-Bewegung. Entstehung, Entwicklung und Nutzen. Berlin: Wissenschaftlicher Verlag.

Latour, Bruno (2010): The Making of Law. An Ethnography of the Conseil d'Etat. Cambridge: Polity Press.

McVeigh, Joseph (2012): „Nachwort“, in: Bachmann, Ingeborg: Die Radiofamilie. Hg. von ders. Berlin: Suhrkamp, S. 337-388.

Müller-Dietz, Heinz (2003): „Der Richter und die Wahrheit - Eine Reminiszenz an Ingeborg Bachmanns Erzählung ,Ein Wildermuth“', in: Hermann Weber (Hg.): Reale und fiktive Kriminalfälle als Gegenstand der Literatur. Berlin: BWv, S. 145-156.

Nöhbauer, Hans F. (1991): „Die Dichterin mit Führerschein“, in: Abendzeitung. München. 5./6./7. Januar 1962, S. 8. Wiedergegeben in: Bachmann, Ingeborg: Wir müssen wahre Sätzen finden. Gespräche und Interviews. Hg. von Christine Koschel und Inge von Weidenbaum. München u.a.: Piper, S. 30-34.

Pilipp, Frank (2001): Ingeborg Bachmanns „Das dreißigste Jahr“. Kritischer Kommentar und Deutung. Würzburg: Königshausen \& Neumann.

Posner, Richard (1988): Law and Literature. Cambridge/Mass.: Harvard University Press.

Schneider, Jost (2013): „Das dreißigste Jahr und Erzählfragmente aus dem Umfeld“, in: Monika Albrecht/Dirk Göttsche (Hg.): Bachmann Handbuch. Leben - Werk Wirkung. Sonderausgabe. Stuttgart/Weimar: J.B. Metzler, S. 112-126.

Schüttpelz, Erhard (2006): „Die medienanthropologische Kehre der Kulturtechniken“, in: Archiv für Mediengeschichte, S. 121-135.

Serres, Michel (1994): Der Naturvertrag. Frankfurt a.M.: Suhrkamp.

Siegert, Bernhard (2001): „Kakographie oder Kommunikation? Verhältnisse zwischen Kulturtechnik und Parasitentum“, in: Archiv für Mediengeschichte, S. 87-99.

Simons, Oliver/Weber, Elisabeth (2008): Bachmanns Medien. Berlin: Vorwerk 8.

Solibakke, Karl Ivan (2012): „,Fest steht der Schrei. Zur Krise der Wahrheit in Ingeborg Bachmanns Ein Wildermuth“, in: Karl Ivan Solibakke/Karina von Tippelskirch (Hg.): Die Waffen nieder! Lay down your weapons! Ingeborg Bachmanns Schreiben gegen den Krieg. Würzburg: Könighausen \& Neumann, S. 225-244.

Steinhauer, Fabian (2015): Vom Scheiden. Geschichte und Theorie einer juristischen Kulturtechnik. Berlin: Duncker \& Humblot.

Töller, Ursula (1998): Erinnern und Erzählen. Studien zu Ingeborg Bachmanns Erzählband „Das Dreißigste Jahr“. Berlin: Erich Schmidt.

Vismann, Cornelia (2011): Medien der Rechtsprechung. Hg. von Alexandra Kemmerer und Markus Krajewski. Frankfurt a.M.: Fischer.

Weigel, Sigrid (1999): Ingeborg Bachmann. Hinterlassenschaften unter Wahrung des Briefgeheimnisses. Wien: Paul Zsolnay. 